\title{
Review on modern techniques in diagnostic and planning radiology
}

\begin{abstract}
Background: The paper contains a brief comprehensive review of new perspective techniques for the cancer detection and treatment in modern diagnostic radiology including stereotactic irradiation, total body irradiation, total skin electron irradiation, intra operative radiotherapy, endocavitary rectal irradiation, conformal radiotherapy, image guided radiotherapy, adaptive radiotherapy, respiratory gated radiotherapy, PET/CT scanners and PET/CT image fusion approaches. The image acquisition, recognition and analyses proper to different techniques, radiation sources, doses and treatment strategies for them, as well as advantages and disadvantages are discussed.
\end{abstract}

Keywords: medical diagnostics, cancer, radiology, image analyses
Volume 4 Issue 6 - 2018

\author{
Natalya Kizilova \\ Warsaw University of Technology, Poland
}

Correspondence: Natalya Kizilova, Institute of Aeronautics and Applied Mechanics, Warsaw University of Technology, Nowowiejska st., 24, 0-665, Poland, Tel: +48 22234 7444, Email n.kizelova@gmail.com

Received: October 05, 2018 | Published: November 02, 2018

\section{Introduction}

Radiation therapy or radiotherapy (RT or XRT) is the therapy of a series of diseases with ionizing radiation, mostly for cancer treatment by killing malignant cells by X-rays. The X-ray RT could be combined with brachytherapy and irradiation by photon or electron beams. Brachytherapy is delivered by placing the radiation source(s) inside or next to the area that requires treatment. It is widely used as an effective treatment for cervical, prostate, breast, skin cancer and some others. History of the X-ray discovery and their applications in medical X-ray imaging and the cancer treatment is discussed in many books and textbooks. ${ }^{1-3}$ Radiation therapy acts on the affected tissues and malignant cells by direct damaging of their DNA or indirect damage via ionization of their atoms and water molecules, promoting the free hydroxyl radicals formation. The photon therapy acts by free radical formation. Since the cancer cells possess lower ability to repair the cellular damage in comparison with healthy ones, and the radiation damage accumulates in the cancer cells gradually leading to their death or, at least the much lower reproducibility. Heavy charged particles like protons, boron, carbon, and neon ions cause significant damage to double- stranded DNA of the cancer cells by direct high energy transfer. Those particles produce small side scatter to the healthy tissue due to their large masses. Nowadays combination of the X-ray, photon and heavy particle beams is a promising developing technology. ${ }^{4-6}$ Contemporary methods of the treatment/surgery planning and control over the therapy/rehabilitation processes are based on precise visualization and digitization of the target region not only in the radiation oncology, but in all areas of modern medicine. ${ }^{3,7-9}$ Tremendous growth of computer technologies and methods of image acquisition and analyses influenced rapid development of new approaches allowing more precise targeting of the malignant tissues, dose calculation, treatment planning and the dose delivery. A brief comprehensive review of new perspective techniques for the cancer detection and treatment in modern diagnostic radiology is presented in this article.

\section{Imaging techniques for special target localization in radiotherapy}

Special target localization techniques in radiotherapy include stereotaxy, image guided radiotherapy, respiratory guided radiotherapy, adaptive radiotherapy, and fusion of positron emission tomography (PET), computed tomography (CT), magnetic resonance imaging (MRI) and ultrasound (US). ${ }^{1,4-6}$

\section{Stereotactic irradiation}

Stereotactic irradiation (SI) is focal irradiation techniques that use multiple, non-coplanar photon beams to deliver a preliminary calculated and prescribed dose of IR to pre-selected lesions, in the brain or other parts of the body. During the past 20 years SI is developed from obscure body irradiation to a mainstream radiotherapy technique that is practiced in the radiotherapy centers all over the world. In SI the prescribed dose can be delivered with (1) one or several external radiation sources (stereotactic external beam irradiation) or through implantated radioactive source(s) among which could be the stereotactic brachytherapy, temporary or permanent implant(s). ${ }^{10}$

High dose rate brachytherapy (HDB) uses a special HDR unit. In the case of the prostatic cancer, with trans rectal US a template as a guide and a set of metal needles are introduced into the prostate. Then a template is sutured to the screen between the anus and scortum, and the metal needles are replaced by hollow plastic ones. A thin HDR cable passes through the needles and deliver high-intensity radiation directly to the prostatic cancer. ${ }^{11}$ The SI technique of combined use of stereotaxy and irradiation was developed by Lars Leksell (Stockholm, Sweden) in 1951 using ortho voltage X-rays. L.Leksell also introduced the term "radiosurgery" to describe the technique of SI with a high single dose. The stereotaxic technique enables the accurate insertion of the needle electrode into any determined area occupied by the cancer cells and its destruction by electro-coagulation or electrolysis. ${ }^{12,13}$ Then the needle was replaced by narrow beams of radiant energy directed at the target in impaired organ and, in that way, produce local destruction(s) of the cancer cells and affected tissues. ${ }^{14}$ In the late 1950s L.Leksell introduced into the radiosurgery the high-energy proton beams from a cyclotron. The technique is still in use today, but is not widespread because of the large operating costs of cyclotrons and their availability. Total prescribed doses in this technique are $\sim 10-50 \mathrm{~Gy}$. The planned targets are commonly small, with typical volumes $\sim 1-35 \mathrm{~cm}^{3}$. The technique needs high positional accuracy for the dose delivery to the target and numerical accuracy in the preliminary computation of the dose needed. It is essentially, any radiation beam that has been found useful for external beam 
radiotherapy has also found its use in radiosurgery, for instance, ${ }^{60} \mathrm{Co}$ gamma rays, megavoltage $\mathrm{X}$-rays, proton and heavy charged particle beams, and neutron beams. There are two modalities of SI: stereotactic radiosurgery (SRS) and stereotactic radiotherapy (SRT). ${ }^{1,4,5}$ SRS is a technique of delivering a single high dose of radiation to the confined, well-defined small PTV. It almost avoids healthy tissues and sensitive organs nearby. SRT is based on the same dose delivery techniques in multiple to either localized or distributed target. Clinical requirements for SI include, first of all, accurate determination of the total dose needed and the fractionation scheme required for the treatment of a particular disease. Then the accurate positional and numerical delivery of the dose to the predetermined target is essential. ${ }^{15}$ The SI technique strictly demands a low skin dose for avoiding epilation, and low eye lens dose for avoiding cataract formation, and low or negligible scatter and leakage dose to the radiosensitive organs for avoiding subsequent undesirable somatic and genetic effects. SI technic is used for treatment of the functional disorders like trigeminal neuralgia, Parkinson's disease, epilepsy, intractable pain, psychoneurosis; vascular lesions (arteriovenous malformation, acoustic neuroma, cavernous angioma, arterial aneurism), primary benign tumours (pituitary adenoma, meningioma, chordoma, craniopharyngioma, meningioblastoma), primary malignant tumours (glioblastoma multiforme, pineal tumour, medulloblastoma, lymphoma), and metastatic tumours. ${ }^{1,4,5}$

\section{Image analyses for SI}

SI uses the image analysis techniques allowing determination the space coordinates of the target tissues in a given fixed system of coordinates..$^{7-9,16}$ In the simplest case the cartesian coordinates of a head can be determined by the rectangle frame (Figure 1A) supplied with special fiducial marks (Figure 1C). The determination technique varies from the manual till Magnetic resonance imaging (MRI) and computed tomography $(\mathrm{CT})$ with the marks tightly connected to the characteristic anatomical features of a head (Figure 1C) in three space projections. Then location of any detected target can be digitized in the 3D space coordinates. The original Leksell stereotactic frame with surgical attachments is presented in Figure 1D. Based on the digitized data, accurate determination of the target volume and its location is determined and the 3D dose distributions inside and outside the target are calculated. The dose-volume histograms (DVHs) for the target and for the specific sensitive organs nearby the target are also computed. The dose distributions that conform to the target shape and give a sharp dose fall-off outside the target volume are needed for the successful treatment. Direct superposition of the isodose distributions on the CT/ MRI images shows the anatomical location of the target according to its digitized location. As sources for RT the ortho voltage X-rays from the X-ray machines, neutron beams from cyclotrons, protons and heavy ions from cyclotrons and synchrotrons are used. Recently the popular Gamma Knife technique has widely used. ${ }^{16,17}$ It is based on 201 gamma ray beams each with activity $1.1 \mathrm{TBq}$ (30Ci) and average distance $40 \mathrm{~cm}$. Gamma Knife was originally designed by Leksell in 1968 and used 179 sources of ${ }^{60} \mathrm{Co}$. Also megavoltage $\mathrm{X}$-rays from Linacs (linear acceleration) systems with multiple converging arcs (Figure 2) and dynamic rotation $360^{\circ}$ are used in the radiosurgery from mid 1980s. The Gamma Knife is shaped as a helmet (Figure $3 \mathrm{~A}$ ) and the sources inside it are adjusted to provide the intersection of the beams in the isocentric point (Figure 3B). During to 50 years of evolution, from 1968 to 2018, Gamma knife has been significantly modified. ${ }^{18-21}$ Modern systems are based on the smaller helmet with additional collimators, while the ${ }^{60} \mathrm{Co}$ sources are located in the outer stationary system supplied with a shelter.

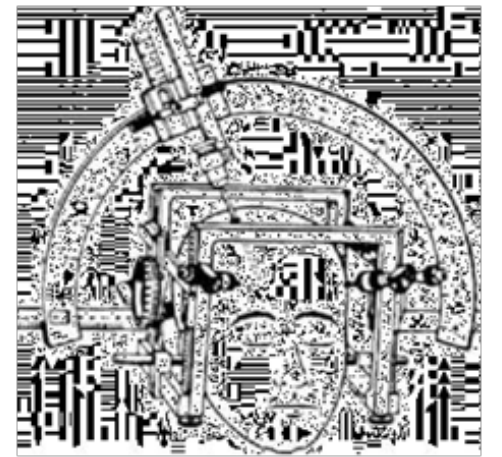

A

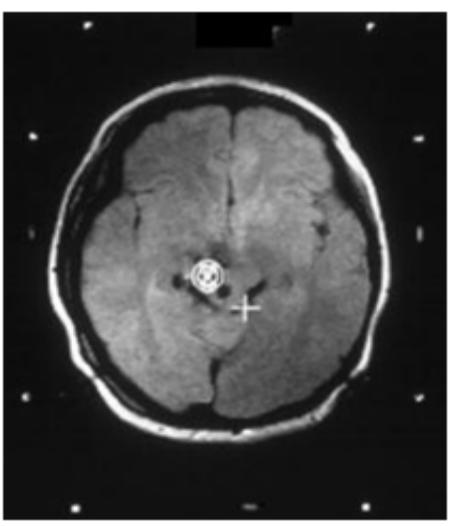

C

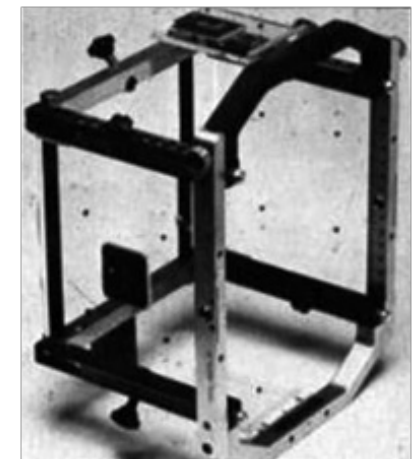

$\mathrm{B}$

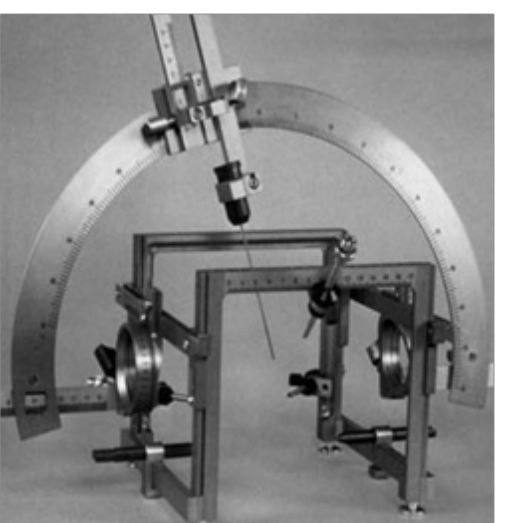

$\mathrm{D}$

Figure I (A) Stereotactic frame with a head, (B) target localization box with fiducial marks, (C) CT slice of a head (white spots are fiducial marks) and (D) original Leksell stereotactic frame with surgical attachments 


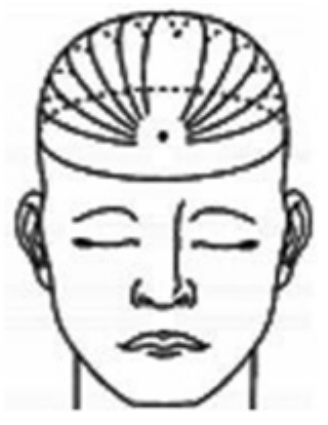

A

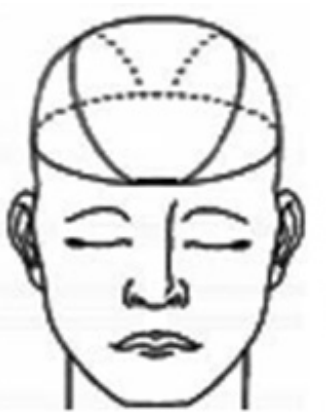

B

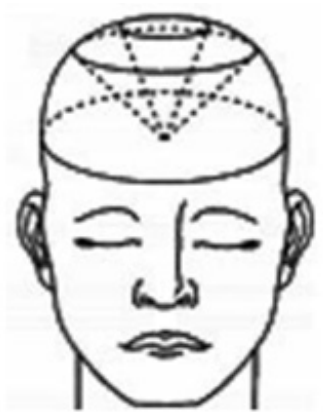

$\mathrm{C}$

Figure 2 (A)Multiple non-coplanar converging arcs technique: multiple converging archs, (B) dynamic rotation, (C) conical rotation.
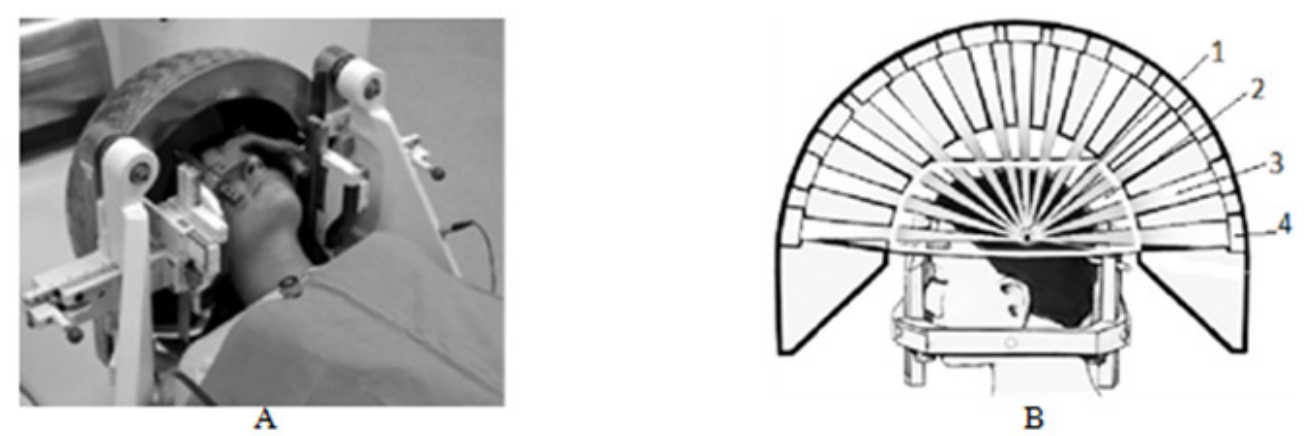

Figure 3 (A) Gamma Knife on a head: $3 d$ and (B) $2 d$ view; I is the target, 2 is the helmet, 3 is the beam, 4 is the ${ }^{60}$ Co source.

\section{Radiation sources for $\mathbf{S I}$}

Therefore, SI technique is based on the stereotactic which defines a fixed Cartesian coordinate system for an accurate localization and further irradiation of the planned target volume (PTV). Imaging equipment like CT, MRI, or digital subtraction angiography (DSA) for lesions and PTVs visualization are used for determination and localization of the PTV and specific sensitive organs around it. Different target localization software is used for determination the coordinates of the target in the stereotactic reference system of coordinates. The treatment planning graphical system is used for calculations of the 3D dose distribution and its superimposition onto the individual anatomical images. At last, an appropriate radiation source and radio surgical treatment technique must be chosen for the patient-individual radiotherapy.

Linac based stereotactic radiosurgery may be used in the form of

a) Conical rotation (Figure 2C): isocentric $\operatorname{Linac}^{22}$ and special treatment chair; ${ }^{23}$

b) Cyber knife: miniature Linac on robotic arm, used since mid$1990 \mathrm{~s} ;{ }^{24,25}$

c) Tomotherapy: miniature Linac on CT gantry. ${ }^{26,27}$

Most of the Linac-based radiosurgery techniques use circular radiation beams produced by special collimators attached to the Linac's head. The circular beams usually have diameters $\sim 10-40 \mathrm{~mm}$ in the isocentre and are produced by $10 \mathrm{~cm}$ thick lead cylinders supplied by the circular holes drilled along their axes. Using the original rectangular Linac collimators with small is not recommended. The multiple non-coplanar converging arcs technique provides delivering of the pre computed dose through a series of gantry arcs; each arc has a different stationary position relating to the treatment couch (supine position) or chair (sitting position). The angles of the arc are usually $<180^{\circ}$ that is helpful in avoiding the parallel-opposed beams in the plane of the arc. Commonly, the number of arcs is from 4 to 11. The main feature of the dynamic rotation technique is the couch based frame approach and the permanent simultaneous rotation of the gantry and couch during the treatment. The rotational angles of the gantry and the coach are $30^{\circ}-330^{\circ}$ and $-75^{\circ}-+75^{\circ}$. The dynamic rotation provides similar dose fall-offs outside the PTV as the multiple non-coplanar converging arcs and the Gamma Knife techniques do. A comparison of Gamma Knife and isocentric Linac is given in Table 1. New developments in the Linac-based radiosurgery includes fractionated SRT, that is better suited for treatment of malignant disease that the conventional SRS. Treatment with irregular fields produced by micro MLCs improves the target dose homogeneity in contrast to multiple isocentre technique implemented in the Gamma Knife. Another development is connected to application of very small radiation fields with $\mathrm{d} \sim 1 \mathrm{~mm}$ which are available on Linacs in contrast to the minimum $\mathrm{d} \sim 4 \mathrm{~mm}$ available with a Gamma Knife. A promising development of SRS is connected with relocatable frames and frameless radiosurgery with Linacs.

Table I Comparative parameters of Gamma Knife and isocentric Linac

\begin{tabular}{lll}
\hline & Gamma knife & Isocentric linac \\
\hline Radiation beam & Stationary & Stationary or movable \\
Radiation quality & 20I sources of ${ }^{60} \mathrm{Co}$ & X-rays 6-18 MV \\
Type of fields & Circular & $\begin{array}{l}\text { Circular or irregular } \\
\text { produced with micro MLC }\end{array}$ \\
Field diameter & $4,8,14,18 \mathrm{~mm}$ & $5-40 \mathrm{~mm}$ \\
Irregular field & - & - \\
Operation & Dedicated & Used for standard RT \\
\hline
\end{tabular}




\section{Targeting techniques in radiosurgery}

Shape and structure of the target are essential for the pre -treatment estimations and decision making. Usually the targets are classified as spherical and irregular ones. For the spherical target (1) the circular field produced by a single spherical isocenter set of beams, (2) Gamma Knife and (3) Linac-based radiosurgery are commonly prescribed. For the irregular target the more complex treatment approaches are needed:

i. Circular fields and multiple isocenters - Conformal radiosurgery;

ii. Gamma Knife and Linac-based radiosurgery;

iii. Irregular uniform intensity fields produced by conformal radiosurgery;

iv. Irregular intensity modulated fields produced by intensity modulated radiosurgery;

v. Linac equipped with micro-multi leaf collimator (micro-MLC).

The micro-MLC technique has a series of advantages compared to the multiple isocentre approach. First of all, it provides an improved dose homogeneity inside the target area(s) and a sharper dose fall-off outside the target producing a uniform influence of the affected tissues and sharp decrease of the dose in the healthy tissues outside. ${ }^{28}$ The micro-MLC allows less time-consuming treatment planning, simpler treatment and shorter the treatment time, significantly lower scatter of the beams and leakage of the dose to the patient. ${ }^{29}$ The next step after the targeting is planning of the 3-D treatment by dynamic stereotactic radiosurgery based on a slices of $\mathrm{CT}$ images. Conventionally the benign and metastatic diseases are treated in a single session of stereotactic radiosurgery, while the primary malignant diseases are treated with fractionated regimes of stereotactic radiotherapy. Treatment with Gamma Knife is usually carried out in a single session, while the treatment with linac-based techniques may be delivered in either a single session or multiple sessions. Typical doses for the single session are 12-25Gy; for multiple sessions- 6 times of $7 \mathrm{~Gy}$ with total dose $42 \mathrm{~Gy}$ and the treatment given every second day, or 10 times of $4 \mathrm{~Gy}$ with total dose $40 \mathrm{~Gy}$ and daily treatment. The target dose homogeneity is essential for successful radiotherapy, and in a ideal case it is within of the prescribed dose. ${ }^{30}$ For radiosurgery, the dose conformity to a small PTV of primary malignant tissues is more important than the target dose homogeneity. ${ }^{31}$ Different techniques, mathematical approaches and software are used for 3-D treatment plan for dynamic stereotactic radiosurgery. ${ }^{30-32}$ The criteria for the choice of the treatment plan are grounded on target coverage with various isodose surfaces. In some cases the $50 \%$ isodose surface covers the target well but also irradiates much of healthy surrounding tissue. If the dose is prescribed to the $50 \%$ isodose surface, the target dose in homogeneity is quite large, but the dose conformity to the target is optimal.

\section{Total Body Irradiation (TBI)}

TBI is a radio therapeutic technique that delivers a dose to a patient's whole body in a uniform way by megavoltage photon beams, ${ }^{60} \mathrm{Co}$ gamma rays or megavoltage X-rays. TBI techniques can carry out irradiation of the whole body, part of the body or total nodal irradiation. TBI is primarily used as a part of preparatory cyto reductive conditioning treatment prior to bone marrow transplantation (BMT). For the TBI procedure a realistic, patient-specific phantom should be irradiated and the absolute dose must be evaluated for different positions of the ionization chamber according to the phantom. The phantom approach allows verification of the beam output calibration and the whole dosimetry chain used in the TBI treatment. As phantoms the water equivalent slab or cylindrical phantoms made of solid water, polystyrene or Lucite for the measurements with stationary electron beams. Anthropomorphic body and head phantoms are also used for measurements with clinical beams. The dose uniformity at the maximal depth in a water equivalent phantom must be at least $80 \%$ of the central nominal field area. ${ }^{33}$ Contemporary TBI techniques use megavoltage photon beams produced by ${ }^{60} \mathrm{Co}$ sources or Linacs. The beams could be stationary with field sizes $\sim 70-\mathrm{x} 200 \mathrm{~cm}^{2}$ applied to the whole body of the patient, or translationally or rotationally moving to cover the whole body with smaller field sizes. Usually, the parallelopposed irradiations are used by delivering the preplanned dose via two equal installations simultaneously or via one installation by switching the patient's position (from supine to inverse).$^{34}$ Different doses are used in TBI. High doses are used as adjuvant therapy in treatment of Ewing's sarcoma and non-Hodgkin's lymphoma, with dose delivery in a single session 750-900cGy or in up to six fractions of $200 \mathrm{cGy}$ each in 3 days with total dose of $1200 \mathrm{cGy}$. The low dose TBI is usually used in the treatment of neuroblastoma, lymphoma, lymphocitic leukaemia, with the dose delivered in 10-15 fractions with $10-15 \mathrm{cGy}$ in each of them. During the part -body irradiation, a dose of $8 \mathrm{~Gy}$ is delivered in a single session to the upper or lower half of the body. The total nodal irradiation is conventionally used as adjuvant treatment of autoimmune diseases, with a nodal dose $40 \mathrm{~Gy}$ delivered in 20 fractions. Total skin electron irradiation (TSEI) is a radiotherapy technique allowing irradiation of the whole patient' skin with the prescribed radiation dose while all other organs are sheltered from any appreciable radiation dose. Typical dose for TSEI is $20 \times 40$ Gy, beam energy on the phantom surface is $\sim 4-7 \mathrm{MeV}$, beam energy at the waveguide exit window is $\sim 6-10 \mathrm{MeV}$, and shaded surface display (SSD) $\sim 300-500 \mathrm{~cm}^{2}$. TSEI is used for treatment of generalized skin malignancies like mycosis fungoides. Since the population requiring TSEI is relatively small and the TSEI techniques are relatively complex and cumbersome, the TSEI technique is available in the largest radiotherapy clinics only. In the past though superficial X-rays were used for TSEI, while contemporary procedures use electron Linacs modified for delivery of the large and uniform electron fields delivered to the whole body. Photon contamination of the electron beam, which is harmful for a patient, must be known to ensure that the prescribed electron dose is not accompanied by a harmfully high total body photon dose. Some areas of the patient's skin (pigmentations, moles, birthmarks) and organs (eyes, nails) must be shielded to avoid the health damage. ${ }^{35}$

Contemporary TSEI techniques may be split in three main groups:

a) Translational techniques, when a patient is translated on a stretcher through an electron beam of sufficient width to cover his/her transverse dimensions;

b) Large electron field techniques, when a standing stationary patient is treated at a large SSD with a single large electron beam or a combination of large electron beams;

c) Rotational techniques, when a patient is standing on a rotating platform in a large electron field. Accepted TSEI standards recommend the use of a large and uniform stationary electron field produced by a megavoltage linac using a suitable electron beam at an extended SSD. 


\section{Intraoperative radiotherapy}

Intraoperative radiotherapy (IORT) is a radio therapeutic technique to deliver a radiation dose of $\sim 10-20$ Gy to a surgically exposed internal organ or tumour(s) in a single session. IORT combines surgery and radiotherapy. Usually an IORT team consists of a surgeon, radiation oncologist, medical physicist, pathologist, radiation therapist, anesthesiologist, and nurse. IORT is usually delivered by ortho voltage X-rays, megavoltage electrons, high dose rate brachytherapy with ${ }^{192}$ Ir source. Mostly the gastrointestinal (rectum, pancreas, stomach), retroperitoneum, bladder, breast and gynecological cancers are treated with IORT, but in general, any tumour site which can be exposed surgically and isolated from the surrounding radiationsensitive tissues/organs can be treated with the IORT. Commonly a large radiation dose is delivered during the surgical procedure in order to improve the local and regional control over the treatment. The advantage of the IORT is in ability to define the target area in situ and decrease the radiation damage of healthy tissues and radiation sensitive organs by direct shielding of them. The disadvantage of the IORT is in the possibility of only single session dose delivery.

\section{D Conformal radiotherapy}

In the past, radiation dose and distribution of beams were computed based on the height, width and depth of the target, so the neighboring healthy tissues were also exposed to radiation. New technologies allow a cancer treatment that shapes the radiation beams to match the shape of the tumor. This approach called 3D conformal radiation therapy relies on multiple beams with optimized intensity modulated fluency distributions. The approach based on the intensity modulated radiotherapy (IMRT) came from mid-1990s. The IMRT dose delivery is possible with tomotherapy unit or isocentric Linac in combination with a multi leaf or MIMIC collimator. The leaves of the collimator can be separately driven by a miniature DC motor. IMRT treatment needs special procedure of the treatment planning. The 3D anatomic data must be multilateral, i.e. the image data from CT or/and MRI in combination with positron emission tomography (PET), single photon emission computed tomography (SPECT) or DSA, depending on the disease. PET is an imaging technique that given not static anatomical but dynamical functional data on the state of the organ(s) based on the intensity of the metabolic processes in it. PET detects pairs of $\gamma$-rays emitted by a positron-emitting radionuclides like fluorine-18, oxygen-15, carbon-11, etc. which are introduced into the organism on molecular tracers. The 3D images of tracer concentration reflect specific activity in the region. The PET popular tracer fludeoxyglucose is an analogue of glucose and its concentration reflects local metabolic activity which is connected to the glucose uptake. In clinical oncology another glucose analog fluorine-18 (fluorodeoxyglucose) is used. It has exceptionally long half-time (110min). SPECT is a tomographic imaging technique with $\gamma$-rays usage. It is based on injection into the bloodstream of the patient a gamma-emitting radioisotope attached to a specific ligand and creating a radio ligand that can be bond to specific type of tissues. In that way, the corresponding 3D image represents the concentration field of the radio ligand (i.e. cells with certain properties) in the region examined. Therefore, both techniques are more essential in the cancer diagnostics and the 3D treatment planning than the traditional anatomic images. The PET and SPECT images allow determination of the intra-tumour regions which require the lower/higher doses for the treatment because of tumor hypoxia, low intrinsic radio sensitivity, or others. Image registration includes fusion of the data sets obtained from different imaging techniques. Image segmentation means slice-by-slice detection and digitization of the anatomic regions of interest, like the anatomic landmarks, gross target volume, organs and tissues at risk, etc. Based on the series of $2 \mathrm{D}$ slices the $3 \mathrm{D}$ model of the target region with the healthy structures nearby can be restored..$^{7-9}$ Using the 3D image, the mostly affected tissues (gross target volume), partially affected (clinical target volume), and at risk (planning target volume) can be localized, and their coordinates, dimensions, volumes and mass can be computed for the dose computations. The differences between the traditional "square" planning and 3D confocal planning, as well as well as difficulties and uncertainties in the determining the tumor, gross, clinical and planning target volumes for the radiotherapy are discussed here. ${ }^{36,37}$

\section{Conclusion}

During the past decades radiation therapy developed from the traditional sources of X-rays to external beam radiotherapy, intensitymodulated radiotherapy, image guided radiotherapy, intensity modulated radiotherapy, rotational IMRT, stereotactic ablative radiotherapy/radiosurgery. The retrospective analysis of the more than 100 years of the X-ray usage for the treatment of tuberculosis, cancer and other diseases as it was reported in this review article, helps in understanding the perspectives and new directions of its development based on new technologies. Recent advances in high precision radiotherapy of cancer and other diseases are based on the novel technology of target localization (image acquisition and segmentation, multimodality fusion, virtual simulation), in silico treatment planning, precise way for the dose delivery (IMRT, tomotherapy, Cyber Knife). Future development of radiation medicine will be connected with improvement of the image analysis based determination of the geometry, anatomy and functional state of the target region, computerbased modeling of the radiation therapy/surgery with the risks and outcomes estimation, and precise space and time delivery of the dose to the target region.

\section{Acknowledgements}

Author is grateful to the organizers and participants of the TAIEX Regional Workshop "Biomedical Engineering - Medical Physics" organized in co-operation with Ministry of Civil Affairs of Bosnia and Herzegovina in Tuzla, Bosnia and Herzegovina, 25-26 June 2018 for the interest to the work and stimulating discussions.

\section{Conflict of interest}

Authors declare that there is no conflict of interest.

\section{References}

1. Morton ER. A text-book of radiology (X-rays). St Louis CV Mosby Company; 1919.

2. Newberry SP. History of X-ray Microscopy. X-ray Microscopy. 1987:346-360.

3. Russo P. Handbook of X-ray Imaging: Physics and Technology. CRC Press; 2018.

4. Haas OC. Radiotherapy Treatment Planning: New System Approaches. London; Springer: 1998.

5. Podgorsak EB. Radiation Oncology Physics: A Handbook for Teachers and Students. Vienna; 2005. 
6. Willett CG, Czito BG, Tyler DS. Intraoperative Radiation Therapy. $J$ Clinical Oncol. 2007;25(8):971-977.

7. Taylor J. Imaging in Radiotherapy. London (UK): Croom Helm; 1988.

8. Blake MA, Kalra MK. Imaging in Oncology. NY: Springer Science \& Business Media; 2008

9. Gouliamos AD, Andreou J, Kosmidis P. Imaging in Clinical Oncology. NY: Springer Science \& Business Media; 2013.

10. Hoskin P, Coyle C. Radiotherapy in Practice - Brachytherapy. Oxford OUP; 2011.

11. Dutta SW, Alonso CE, Libby B, et al. Prostate cancer high dose-rate brachytherapy: review of evidence and current perspectives. Expert Rev Med Devices. 2018;15(1):71-79.

12. Spiegel EA, Wycis HT, Marks M, et al. Stereotaxic apparatus for operations on the human brain. Science. 1947;106(2754):349-350.

13. Schurr PH, Merrington WR. Horsley-Clarke stereotaxic apparatus. BJS 1978;65(1):33-36.

14. Leksell L. The stereotaxic method and radiosurgery of the brain. Acta Chir Scand. 1951;102(4):316-319.

15. Ding GX, Duggan DM, Coffey CW. Commissioning stereotactic radiosurgery beams using both experimental and theoretical methods. Phys Med Biol. 2006;51(10):2549-2566.

16. DE Heron, M Saiful Huq. Stereotactic Radiosurgery and Stereotactic Body Radiation Therapy. Demos Medical; 2018.

17. Araki F. Monte Carlo study of a Cyberknife stereotactic radiosurgery system. Med Phys. 2006;33:2955-2963.

18. Ganz JC. Gamma Knife Surgery. Springer; 1997.

19. Lunsford LD, Kondziolka D, Flickinger DC. Gamma Knife Brain Surgery. Karger;1998.

20. Germano IM. Linac and Gamma Knife Radiosurgery. Thieme; 2000.

21. Liščák R. Gamma Knife Radiosurgery. Nova Sci Publ Inc; 2013.

22. Chung HT, Park WY, Kim TH, et al. Assessment of the accuracy and stability of frameless gamma knife radiosurgery. J Appl Clin Med Phys. 2018;19(4):148-154.

23. Mc Ginley PH, Butker EK, Crocker IR, et al. An adjustable collimator for stereotactic radiosurgery. Phys Med Biol. 1992;37(2):413-420.

24. McGinley PH, Butker EK, Crocker IR, et al. A patient rotator for stereotactic radiosurgery. Phys Med Biol. 1990;35(5):649-658.
25. Cmelak AJ, Cox RS, Adler JR, et al. Radiosurgery for skull base malignancies and nasopharyngeal carcinoma. Int J Radiat Oncol Biol Phys. 1997;37(5):997-1003.

26. Lo SS, Teh BS, Lu JJ, et al. Stereotactic Body Radiation Therapy. Germany: Springer Sci \& Business Media; 2012.

27. Ruchala KJ, Olivera GH, Schloesser EA, et al. Megavoltage CT on a Tomotherapy System. Phys Med Biol. 1999;44(10):2597-2621.

28. Olivera GH, Shepard DM, Ruchala KJ, et al. Tomotherapy in Modern Technology of Radiation Oncology. In: Van Dyk J, Editor. Madison; Med Phys Publ: 1999.

29. Cosgrove VP, Jahn U, Pfaender M, et al. Commissioning of a micro multi-leaf collimator and planning system for stereotactic radiosurgery. Radiother Oncol. 1999;50(3):325-336.

30. Belec J, Patrocinio H, Verhaegen F. Development of a Monte Carlo model for the Brainlab microMLC. Phys Med Biol. 2005;50(5):787-799.

31. Haridass A, Maclean J, Chakraborty S, et al. Dynamic CT angiography for cyberknife radiosurgery planning of intracranial arteriovenous malformations: a technical/feasibility report. Radiol Oncol. 2015;49(2):192-199.

32. Soisson ET, Hoban PW, Kammeyer T, et al. A technique for stereotactic radiosurgery treatment planning with helical tomotherapy. Med Dosim 2011;36(1):46-56.

33. Yu C, Shepard D. Treatment planning for stereotactic radiosurgery with photon beams. Technol Cancer Res Treat. 2003;2(2):93-104.

34. Sanchez-Doblado F, Andreo P, Capote R, et al. Ionization chamber dosimetry of small photon fields: a Monte Carlo study on stopping power ratios for radiosurgery and IMRT beams. Phys Med Biol. 2003;48(14):2081-2099.

35. Paskalev KA, Seuntjens JP, Patrocinio HJ, et al. Physical aspects of dynamic stereotactic radiosurgery with very small photon beams ( 1.5 and $3 \mathrm{~mm}$ in diameter). Med Phys. 2003;30(2):111-118.

36. Li S, Rashid A, He S, et al. A new approach in dose measurement and error analysis for narrow photon beams (beamlets) shaped by different multileaf collimators using a small detector. Med Phys. 2004;31(7):2020-2032.

37. Burnet NG, Simon J Thomas, Kate E Burton, et al. Defining the tumour and target volumes for radiotherapy. Cancer Imag. 2004;4(2):153-161. 